J. Korean Math. Soc. 48 (2011), No. 6, pp. 1125-1142

http://dx.doi.org/10.4134/JKMS.2011.48.6.1125

\title{
SEQUENCE SPACES OF OPERATORS ON $l_{2}$
}

\author{
Jitti Rakbud and Sing-Cheong Ong
}

\begin{abstract}
In this paper, we define some new sequence spaces of infinite matrices regarded as operators on $l_{2}$ by using algebraic properties of such the matrices under the Schur product multiplication. Some of their basic properties as well as duality and preduality are discussed.
\end{abstract}

\section{Introduction}

Let $1 \leq p, q<\infty$. For any infinite scalar matrix $A=\left[a_{j k}\right]$, we say that $A$ defines a linear operator from $l_{p}$ into $l_{q}$ if for each $x=\left\{x_{k}\right\}_{k=1}^{\infty}$ in $l_{p}$ the series $\sum_{k=1}^{\infty} a_{j k} x_{k}$ converges for all $j$, and the sequence $A x:=\left\{\sum_{k=1}^{\infty} a_{j k} x_{k}\right\}_{j=1}^{\infty}$ belongs to $l_{q}$. In this circumstance, we call the linear operator $x \mapsto A x$ the linear operator defined by $A$. If $A$ defines a linear operator from $l_{p}$ into $l_{q}$, then by the uniform boundedness principle, the linear operator defined by $A$ is bounded. For each $1 \leq p, q<\infty$, let $\mathcal{B}\left(l_{p}, l_{q}\right)$ be the set of all infinite matrices defining linear operators from $l_{p}$ into $l_{q}$. In the case where $p=q$, we denote $\mathcal{B}\left(l_{p}, l_{p}\right)$ by just $\mathcal{B}\left(l_{p}\right)$. For any matrix $A$, we define $\|A\|_{p, q}$ to be the norm of the linear operator defined by $A$ if $A \in \mathcal{B}\left(l_{p}, l_{q}\right)$, and to be $\infty$ otherwise. It is well known that $\mathcal{B}\left(l_{p}, l_{q}\right)$ equipped with the norm $\|\cdot\|_{p, q}$ is a Banach space. In fact, it is exactly the set of matrix representations of all bounded linear operators from $l_{p}$ into $l_{q}$ with respect to the standard Schauder bases on $l_{p}$ and $l_{q}$. For any matrix $A$, we call $A$ bounded if $A \in \mathcal{B}\left(l_{p}, l_{q}\right)$ and unbounded otherwise. It is known that a matrix $A$ belongs to $\mathcal{B}\left(l_{p}, l_{q}\right)$ if and only if $\sup _{n}\left\|A_{n_{\lrcorner}}\right\|_{p, q}<\infty$, where $A_{n}$ is the matrix whose entries in the upper left $n \times n$-block are exactly those of $A$ and are zero otherwise, and also known that for any matrix $A,\left\|A_{n_{\lrcorner}}\right\|_{p, q} \nearrow\|A\|_{p, q}$. A matrix $A$ is said to be compact if the linear operator defined by $A$ is compact. It is well known that a matrix $A$ is compact as an operator on $l_{2}$ if and only if $\left\|A_{n_{\lrcorner}}-A\right\|_{2,2} \rightarrow 0$, which is equivalent to $\left\|A_{\underline{n}}-A\right\|_{2,2} \rightarrow 0$, where $A_{\underline{n}}$ is the matrix which agrees with $A$ on the first $n$ rows and are 0 on all other entries.

Received August 30, 2009; Revised December 19, 2010.

2010 Mathematics Subject Classification. Primary 47L05; Secondary 15A04.

Key words and phrases. bounded matrix, unbounded matrix, compact matrix. 
The Schur product (also known as the Hardamard product or entry-wise product) of two scalar matrices $A=\left[a_{j k}\right]$ and $B=\left[b_{j k}\right]$ of the same size is the matrix $A \bullet B:=\left[a_{j k} b_{j k}\right]$. In [6], Schur proved that $\mathcal{B}\left(l_{2}\right)$ is a commutative Banach algebra under the operator norm and the Schur product. In [1], G. Bennett extended the result from the study of Schur mentioned above. He proved that for each $1 \leq p, q<\infty, \mathcal{B}\left(l_{p}, l_{q}\right)$ is also a Banach algebra under the Schur product. In [2] P. Chaisuriya and S.-C. Ong studied the class of matrices over any Banach algebra with identity. In that paper, for a fixed Banach algebra $\mathfrak{B}$ with identity and $1 \leq p, q, r<\infty$, the authors defined the class $\mathcal{S}_{p, q}^{r}(\mathfrak{B})$ of matrices $A=\left[a_{j k}\right]$ over $\mathfrak{B}$ such that the absolute Schur rth-power $A^{[r]}:=\left[\left\|a_{j k}\right\|^{r}\right]$ defines a linear operator from $l_{p}$ into $l_{q}$. And they proved that it is a Banach algebra under the the absolute Schur $r$-norm defined by $\|A\|_{p, q, r}=\left\|A^{[r]}\right\|_{p, q}^{1 / r}$ and the Schur product which is generalized to this setting via the product in the Banach algebra $\mathfrak{B}$. The authors also gave a nice relationship between the class $\mathcal{B}\left(l_{p}, l_{q}\right)$ of all bounded operators and the algebra $\mathcal{S}_{p, q}^{2}(\mathbb{C})$. They found that $\mathcal{S}_{p, q}^{2}(\mathbb{C})$ contains $\mathcal{B}\left(l_{p}, l_{q}\right)$ as a non-closed ideal.

In this paper, we provide some reasonable ways to define sequence spaces of matrices in the Banach algebra $\mathcal{S}_{2,2}^{2}(\mathbb{C})$ which from the results in [2] mentioned above contains all bounded matrices (or operators on $l_{2}$ ) as an ideal and also contains some unbounded ones. The pleasant structure of the algebra $\mathcal{S}_{2,2}^{2}(\mathbb{C})$ may provide us with a reasonable way of studying simultaneously bounded and unbounded matrices (as operators on $l_{2}$ ) in these new settings sequentially.

\section{Definitions and some basic properties}

In this section we introduce some new sequence spaces of matrices and discuss some of their basic properties. Before going to the goal, we need some preliminary results about the Banach algebra $\mathcal{S}_{2,2}^{2}(\mathbb{C})$. For convenience, we denote here the Banach algebra $\mathcal{S}_{2,2}^{r}(\mathbb{C})$ for $r=1,2$ by just $\mathcal{S}^{r}$, the norm $|\|\cdot\||_{2,2,2}$ on $\mathcal{S}^{2}$ by $\mid\|\cdot\| \|_{2}$, the norm $\|\cdot\|_{2,2}$ on $\mathcal{B}\left(l_{2}\right)$ by $\|\cdot\|$, and the matrix $A^{[1]}$ by $|A|$.

The following Cauchy-Schwarz-type inequality was first introduced in [5] by S.-C. Ong and extended in [2].

Theorem 2.1 (Cauchy-Schwarz-type inequality). For any infinite scalar matrices $A$ and $B,\||A \bullet B|\| \leq\left|\|A\|\left\|_{2} \mid\right\| B \|_{2}\right.$.

From the Cauchy-Schwarz-type inequality, the following proposition which is useful for the paper is obtained.

Proposition 2.2. $\mathcal{S}^{2} \bullet \mathcal{S}^{2}:=\left\{A \bullet B: A, B \in \mathcal{S}^{2}\right\}=\mathcal{S}^{1}$.

Proof. If $A, B \in \mathcal{S}^{2}$, then by Theorem 2.1, $A \bullet B \in \mathcal{S}^{1}$. If $A=\left[a_{j k}\right] \in \mathcal{S}^{1}$, then both $B=\left[\left|a_{j k}\right|^{1 / 2}\right]$ and $C=\left[\left|a_{j k}\right|^{1 / 2} e^{i\left(\arg \left(a_{j k}\right)\right)}\right]$ belong to $\mathcal{S}^{2}$ and $A=$ $B \bullet C$.

The following lemma is also a consequence of the Cauchy-Schwarz-type inequality. 
Lemma 2.3. For any $A, B \in \mathcal{S}^{2}$,

$$
\left\|A^{[2]}-B^{[2]}\left|\left\|\leq\left(\|A\|_{2}+\mid\|B\|_{2}\right) \mid\right\| A-B \|_{2} .\right.\right.
$$

Proof. By the Cauchy-Schwarz-type inequality, we obtain

The proof is complete.

$$
\begin{aligned}
\left\|\left|A^{[2]}-B^{[2]}\right|\right\| & =\|\| A|-| B|| \bullet(|A|+|B|) \| \\
& \leq\||A-B| \bullet(|A|+|B|)\| \\
& \leq\|A-B\|\left\|_{2}|\||A|+|B|\||_{2}\right. \\
& \leq\left(\|A\|_{2}+\mid\|B\|_{2}\right)\|A-B\|_{2} .
\end{aligned}
$$

Proposition 2.4. The map $A \mapsto A^{[2]}$ from $\mathcal{S}^{2}$ into $\mathcal{B}\left(l_{2}\right)$ is continuous.

Proof. Suppose that $A_{n} \rightarrow A$ in $\mathcal{S}^{2}$. Then there exists $M>0$ such that $\|A\| \|_{2} \leq M$ and $\left\|A_{n}\right\|_{2} \leq M$ for all $n$. So, by Lemma 2.3,

$$
\left\|A_{n}^{[2]}-A^{[2]}\right\| \leq\left\|A_{n}^{[2]}-A^{[2]}\right\| \leq 2 M \mid\left\|A_{n}-A\right\|_{2} \rightarrow 0 .
$$

It follows that the map $A \mapsto A^{[2]}$ is continuous.

From the structure of the Banach algebra $\mathcal{S}^{2}$, we can reasonably define spaces of sequences of matrices acting as operators on $l_{2}$ as follows:

$$
\begin{gathered}
\mathcal{O}_{b}=\left\{\left\{A_{k}\right\}_{k=1}^{\infty} \subset \mathcal{S}^{2}: \text { the sequence }\left\{\sum_{k=1}^{n} A_{k}^{[2]}\right\}_{n=1}^{\infty} \text { is bounded in } \mathcal{B}\left(l_{2}\right)\right\} ; \\
\mathcal{O}_{c}=\left\{\left\{A_{k}\right\}_{k=1}^{\infty} \subset \mathcal{S}^{2}: \text { the sequence }\left\{\sum_{k=1}^{n} A_{k}^{[2]}\right\}_{n=1}^{\infty} \text { converges in } \mathcal{B}\left(l_{2}\right)\right\} .
\end{gathered}
$$

In the following theorem, we obtain a simple characterization of the set $\mathcal{O}_{b}$.

Theorem 2.5. (1) A sequence $\left\{\left[a_{j i}^{(k)}\right]\right\}_{k=1}^{\infty}$ belongs to $\mathcal{O}_{b}$ if and only if the matrix $\left[\sum_{k=1}^{\infty}\left|a_{j i}^{(k)}\right|^{2}\right]$ belongs to $\mathcal{B}\left(l_{2}\right)$.

(2) If $\left\{A_{k}=\left[a_{j i}^{(k)}\right]\right\}_{k=1}^{\infty}$ belongs to $\mathcal{O}_{b}$, then

$$
\left\|\left[\sum_{k=1}^{\infty}\left|a_{j i}^{(k)}\right|^{2}\right]\right\|=\sup _{n}\left\|\sum_{k=1}^{n} A_{k}^{[2]}\right\| .
$$

Proof. (1) Suppose that a sequence $\left\{A_{k}=\left[a_{j i}^{(k)}\right]\right\}_{k=1}^{\infty}$ belongs to $\mathcal{O}_{b}$. Then for each $(j, i)$, the series $\sum_{k=1}^{\infty}\left|a_{j i}^{(k)}\right|^{2}$ converges. Let $A=\left[\sum_{k=1}^{\infty}\left|a_{j i}^{(k)}\right|^{2}\right]$, let $M=\sup _{K}\left\|\sum_{k=1}^{K} A_{k}^{[2]}\right\|$, and let $\mathbf{x}=\left\{x_{i}\right\}_{i=1}^{\infty} \in l_{2}$ with $\|\mathbf{x}\|_{2} \leq 1$. Then for each fixed $n$, we have by Minkowsi's inequality for scalar sequences that

$$
\left\|A_{n} \mathbf{x}\right\|_{2}=\left\{\left.\left.\sum_{j=1}^{n}\left|\sum_{i=1}^{n} \sum_{k=1}^{\infty}\right| a_{j i}^{(k)}\right|^{2} x_{i}\right|^{2}\right\}^{1 / 2}
$$




$$
\begin{aligned}
\leq & \left\{\sum_{j=1}^{n}\left(\left.\sum_{i=1}^{n}\left|\sum_{k=1}^{K}\right| a_{j i}^{(k)}\right|^{2}-\sum_{k=1}^{\infty}\left|a_{j i}^{(k)}\right|^{2}|| x_{i} \mid\right)^{2}\right\}^{1 / 2} \\
& +\left\{\sum_{j=1}^{n}\left(\left.\sum_{i=1}^{n}\left|\sum_{k=1}^{K}\right| a_{j i}^{(k)}\right|^{2}|| x_{i} \mid\right)^{2}\right\}^{1 / 2} \\
\leq & \left\{\sum_{j=1}^{n}\left(\left.\sum_{i=1}^{n}\left|\sum_{k=1}^{K}\right| a_{j i}^{(k)}\right|^{2}-\sum_{k=1}^{\infty}\left|a_{j i}^{(k)}\right|^{2}|| x_{i} \mid\right)^{2}\right\}^{1 / 2}+\left\|\left(\sum_{k=1}^{K} A_{k}^{[2]}\right)_{n\lrcorner}\right\| \\
\leq & \left\{\sum_{j=1}^{n}\left(\left.\sum_{i=1}^{n}\left|\sum_{k=1}^{K}\right| a_{j i}^{(k)}\right|^{2}-\sum_{k=1}^{\infty}\left|a_{j i}^{(k)}\right|^{2}|| x_{i} \mid\right)^{2}\right\}^{1 / 2}+\left\|\sum_{k=1}^{K} A_{k}^{[2]}\right\| \\
\leq & \left\{\sum_{j=1}^{n}\left(\left.\sum_{i=1}^{n}\left|\sum_{k=1}^{K}\right| a_{j i}^{(k)}\right|^{2}-\sum_{k=1}^{\infty}\left|a_{j i}^{(k)}\right|^{2}|| x_{i} \mid\right)^{2}\right\}^{1 / 2}+M \text { for all } K .
\end{aligned}
$$

Thus, by taking the limit as $K \rightarrow \infty$, we have for each $n$ that $\left\|A_{n\lrcorner} \mathbf{x}\right\|_{2} \leq M$ for all $\mathbf{x} \in l_{2}$ with $\|\mathbf{x}\|_{2} \leq 1$. It follows that $\left\|A_{n_{\lrcorner}}\right\| \leq M$ for all $n$. Consequently, $A \in \mathcal{B}\left(l_{2}\right)$. The converse is obvious.

(2) It is clear that $\sup _{K}\left\|\sum_{k=1}^{K} A_{k}^{[2]}\right\| \leq\left\|\left[\sum_{k=1}^{\infty}\left|a_{j i}^{(k)}\right|^{2}\right]\right\|$. Suppose to the contrary that $\sup _{K}\left\|\sum_{k=1}^{K} A_{k}^{[2]}\right\|<\left\|\left[\sum_{k=1}^{\infty}\left|a_{j i}^{(k)}\right|^{2}\right]\right\|$. Then there is a positive integer $n_{0}$ and an $\mathbf{x} \in l_{2}$ with $\|\mathbf{x}\|_{2} \leq 1$ such that

$$
\sup _{K}\left\|\sum_{k=1}^{K} A_{k}^{[2]}\right\|<\left\|\left[\sum_{k=1}^{\infty}\left|a_{j i}^{(k)}\right|^{2}\right]_{n_{0\lrcorner}}|\mathbf{x}|\right\|_{2} .
$$

This implies that there exists a positive integer $K_{0}$ such that

$$
\left\|\left(\sum_{k=1}^{K_{0}} A_{k}^{[2]}\right)_{n_{0\lrcorner}}\right\| \leq\left\|\sum_{k=1}^{K_{0}} A_{k}^{[2]}\right\| \leq \sup _{K}\left\|\sum_{k=1}^{K} A_{k}^{[2]}\right\|<\left\|\left(\sum_{k=1}^{K_{0}} A_{k}^{[2]}\right)_{n_{0\lrcorner}}|\mathbf{x}|\right\|_{2},
$$

which is a contradiction. Hence

$$
\sup _{K}\left\|\sum_{k=1}^{K} A_{k}^{[2]}\right\|=\left\|\left[\sum_{k=1}^{\infty}\left|a_{j i}^{(k)}\right|^{2}\right]\right\| \text {. }
$$

The proof is finished.

It is apparent that $\mathcal{O}_{c} \subseteq \mathcal{O}_{b}$. The inclusion is proper, indeed, the sequence of matrices $A_{k}$ with $(k, k)$-entry 1 and all other entries zero belongs to $\mathcal{O}_{b}$ but doesn't belong to $\mathcal{O}_{c}$.

In the following proposition, we give some sufficient conditions for a sequence in $\mathcal{S}^{2}$ to be a member of $\mathcal{O}_{c}$. 
Proposition 2.6. (1) If $\left\{A_{k}\right\}_{k=1}^{\infty}$ is a sequence in $\mathcal{B}\left(l_{2}\right)$ with $\sum_{k=1}^{\infty}\left\|A_{k}\right\|^{2}<$ $\infty$, then $\left\{A_{k}\right\}_{k=1}^{\infty}$ belongs to $\mathcal{O}_{c}$.

(2) If $\left\{A_{k}=\left[a_{j i}^{(k)}\right]\right\}_{k=1}^{\infty}$ is a sequence in $\mathcal{S}^{2}$ such that the matrix $\left[\sum_{k=1}^{\infty}\left|a_{j i}^{(k)}\right|^{2}\right]$ is compact, then $\left\{A_{k}\right\}_{k=1}^{\infty}$ belongs to $\mathcal{O}_{c}$.

Proof. (1) It follows immediately from Schur-Bennett's theorem in $[1,6]: \mathcal{B}\left(l_{2}\right)$ is a Banach algebra under the Schur product.

(2) Let $A=\left[\sum_{k=1}^{\infty}\left|a_{j i}^{(k)}\right|^{2}\right]$, and let $\epsilon>0$. Then by the compactness of the matrix $A$, there is a positive integer $N$ such that $\left\|A_{N_{\lrcorner}}-A\right\|<\frac{\epsilon}{3}$. Since the series $\sum_{k=1}^{\infty}\left|a_{j i}^{(k)}\right|^{2}$ converges for all $1 \leq j, i \leq N$, there is a positive integer $K_{0}$ such that for each $1 \leq j, i \leq N$,

$$
\sum_{k=K}^{\infty}\left|a_{j i}^{(k)}\right|^{2}<\frac{\epsilon}{3 N^{3 / 2}} \text { for all } K \geq K_{0} .
$$

It follows that

$$
\begin{aligned}
\left\|\sum_{k=1}^{K} A_{k}^{[2]}-A\right\| \leq & \left\|A_{N_{\lrcorner}}-\left(\sum_{k=1}^{K} A_{k}^{[2]}\right)_{N\lrcorner}\right\|+\left\|\left(\sum_{k=1}^{K} A_{k}^{[2]}\right)_{N\lrcorner}-\sum_{k=1}^{K} A_{k}^{[2]}\right\| \\
& +\left\|A_{N\lrcorner}-A\right\| \\
\leq & \left\{\sum_{j=1}^{N}\left(\sum_{i=1}^{N} \sum_{k=K}^{\infty}\left|a_{j i}^{(k)}\right|^{2}\right)^{2}\right\}^{1 / 2}+2\left\|A_{N_{\lrcorner}}-A\right\| \\
& <\frac{\epsilon}{3}+\frac{2 \epsilon}{3}=\epsilon \text { for all } K \geq K_{0} .
\end{aligned}
$$

Consequently, $\left\{A_{k}\right\}_{k=1}^{\infty} \in \mathcal{O}_{c}$.

For any $\mathbf{A}=\left\{A_{k}=\left[a_{j i}^{(k)}\right]\right\}_{k=1}^{\infty} \in \mathcal{O}_{b}$, we define

$$
\|\mathbf{A}\|:=\left(\sup _{n}\left\|\sum_{k=1}^{n} A_{k}^{[2]}\right\|\right)^{1 / 2}=\left\|\left[\sum_{k=1}^{\infty}\left|a_{j i}^{(k)}\right|^{2}\right]\right\|^{1 / 2} .
$$

It is obvious that $\left|\left\|A_{k}\right\|_{2} \leq\right|\|\mathbf{A}\|$ for all $k$. In the case where $\mathbf{A} \in \mathcal{O}_{c}$, we have

$$
\sum_{k=1}^{\infty} A_{k}^{[2]}=\left[\sum_{k=1}^{\infty}\left|a_{j i}^{(k)}\right|^{2}\right]
$$

Hence we obtain in this case

$$
\|\mathbf{A}\|=\left\|\sum_{k=1}^{\infty} A_{k}^{[2]}\right\|^{1 / 2} .
$$

From Proposition 2.2, the following Cauchy-Schwarz's inequality for $n$-tuples of matrices in $\mathcal{S}^{2}$ makes sense. 
Theorem 2.7 (Cauchy-Schwarz's inequality). For any two $n$-tuples $\left(A_{1}, A_{2}, \ldots\right.$, $\left.A_{n}\right)$ and $\left(B_{1}, B_{2}, \ldots, B_{n}\right)$ of matrices in $\mathcal{S}^{2}$,

$$
\begin{aligned}
\left\|\sum_{k=1}^{n} A_{k} \bullet B_{k}\right\| \leq\left\|\left|\sum_{k=1}^{n} A_{k} \bullet B_{k}\right|\right\| & \leq\left\|\sum_{k=1}^{n}\left|A_{k} \bullet B_{k}\right|\right\| \\
& \leq\left\|\sum_{k=1}^{n} A_{k}^{[2]}\right\|^{1 / 2}\left\|\sum_{k=1}^{n} B_{k}^{[2]}\right\|^{1 / 2} .
\end{aligned}
$$

Proof. Suppose that $A_{k}=\left[a_{j i}^{(k)}\right]$ and $B_{k}=\left[b_{j i}^{(k)}\right]$ for all $k=1,2, \ldots, n$. Then we have by Cauchy-Schwarz's inequality for scalar sequences and CauchySchwarz's inequality for matrices that

$$
\begin{aligned}
\left\|\sum_{k=1}^{n}\left|A_{k} \bullet B_{k}\right|\right\| & =\left\|\left[\sum_{k=1}^{n}\left|a_{j i}^{(k)} b_{j i}^{(k)}\right|\right]\right\| \\
& \leq\left\|\left[\left(\sum_{k=1}^{n}\left|a_{j i}^{(k)}\right|^{2}\right)^{1 / 2}\left(\sum_{k=1}^{n}\left|b_{j i}^{(k)}\right|^{2}\right)^{1 / 2}\right]\right\| \\
& =\left\|\left[\left(\sum_{k=1}^{n}\left|a_{j i}^{(k)}\right|^{2}\right)^{1 / 2}\right] \bullet\left[\left(\sum_{k=1}^{n}\left|b_{j i}^{(k)}\right|^{2}\right)^{1 / 2}\right]\right\| \\
& \leq\left\|\left[\sum_{k=1}^{n}\left|a_{j i}^{(k)}\right|^{2}\right]\right\|^{1 / 2}\left\|\left[\sum_{k=1}^{n}\left|b_{j i}^{(k)}\right|^{2}\right]\right\|^{1 / 2}\left\|^{1 / 2}\right\|^{1 / 2}\left\|\sum_{k=1}^{n} B_{k}^{[2]}\right\|^{1 / 2} \cdot \\
& =\left\|\sum_{k=1}^{n} A_{k}^{[2]}\right\|^{2}
\end{aligned}
$$

The other two inequalities on the left-hand side are evident, and hence the proof is finished.

From Cauchy-Schwarz's inequality above, the corresponding Minkowski's inequality is obtained.

Theorem 2.8 (Minkowski's inequality). For any two $n$-tuples $\left(A_{1}, A_{2}, \ldots, A_{n}\right)$ and $\left(B_{1}, B_{2}, \ldots, B_{n}\right)$ of matrices in $\mathcal{S}^{2}$,

$$
\left\|\sum_{k=1}^{n}\left(A_{k}+B_{k}\right)^{[2]}\right\|^{1 / 2} \leq\left\|\sum_{k=1}^{n} A_{k}^{[2]}\right\|^{1 / 2}+\left\|\sum_{k=1}^{n} B_{k}^{[2]}\right\|^{1 / 2} .
$$

Proof. From Cauchy-Schwarz's inequality, we have

$$
\left\|\sum_{k=1}^{n}\left(A_{k}+B_{k}\right)^{[2]}\right\|=\left\|\sum_{k=1}^{n} \overline{A_{k}+B_{k}} \bullet\left(A_{k}+B_{k}\right)\right\|
$$




$$
\begin{aligned}
= & \left\|\sum_{k=1}^{n}\left(\overline{A_{k}}+\overline{B_{k}}\right) \bullet\left(A_{k}+B_{k}\right)\right\| \\
= & \left\|\sum_{k=1}^{n} \overline{A_{k}} \bullet\left(A_{k}+B_{k}\right)+\sum_{k=1}^{n} \overline{B_{k}} \bullet\left(A_{k}+B_{k}\right)\right\| \\
\leq & \left\|\sum_{k=1}^{n} \overline{A_{k}} \bullet\left(A_{k}+B_{k}\right)\right\|+\left\|\sum_{k=1}^{n} \overline{B_{k}} \bullet\left(A_{k}+B_{k}\right)\right\| \\
\leq & \left\|\sum_{k=1}^{n} A_{k}^{[2]}\right\|^{1 / 2}\left\|\sum_{k=1}^{n}\left(A_{k}+B_{k}\right)^{[2]}\right\| \|^{1 / 2} \\
& +\left\|\sum_{k=1}^{n} B_{k}^{[2]}\right\|^{1 / 2}\left\|\sum_{k=1}^{n}\left(A_{k}+B_{k}\right)^{[2]}\right\|^{1 / 2} \\
= & \left(\left\|\sum_{k=1}^{n} A_{k}^{[2]}\right\|^{1 / 2}+\left\|\sum_{k=1}^{n} B_{k}^{[2]}\right\|^{1 / 2}\right)\left\|\sum_{k=1}^{n}\left(A_{k}+B_{k}\right)^{[2]}\right\|^{1 / 2},
\end{aligned}
$$

where $\bar{C}:=\left[\overline{c_{j k}}\right]$ for any matrix $C=\left[c_{j k}\right]$. It follows that

$$
\left\|\sum_{k=1}^{n}\left(A_{k}+B_{k}\right)^{[2]}\right\|^{1 / 2} \leq\left\|\sum_{k=1}^{n} A_{k}^{[2]}\right\|^{1 / 2}+\left\|\sum_{k=1}^{n} B_{k}^{[2]}\right\|^{1 / 2} .
$$

The proof is complete.

Now we have the Riesz-Fischer-type theorem.

Theorem 2.9. Both $\mathcal{O}_{c}$ and $\mathcal{O}_{b}$ equipped with the norm $\|\cdot\| \mid$ are Banach spaces.

Proof. From Minkowski's inequality, both $\mathcal{O}_{b}$ and $\mathcal{O}_{c}$ equipped with the norm $\|\cdot\| \mid \|$ are normed spaces. To see that $\mathcal{O}_{b}$ is a Banach space, let

$$
\left\{\mathbf{A}_{n}=\left\{A_{k}^{(n)}\right\}_{k=1}^{\infty}\right\}_{n=1}^{\infty}
$$

be a Cauchy sequence in $\mathcal{O}_{b}$. For each $k$, we have

$$
\left\|A_{k}^{(n)}-A_{k}^{(m)}\right\|_{2} \leq\left\|\mathbf{A}_{n}-\mathbf{A}_{m}\right\| \text { for all } n, m .
$$

This implies that the sequence $\left\{A_{k}^{(n)}\right\}_{n=1}^{\infty}$ is a Cauchy sequence in $\mathcal{S}^{2}$ for all $k$. Thus, by completeness of $\mathcal{S}^{2}$, there is $A_{k}$ in $\mathcal{S}^{2}$ such that $A_{k}^{(n)} \rightarrow A_{k}$. Let $\mathbf{A}=\left\{A_{k}\right\}_{k=1}^{\infty}$, we will show that $\mathbf{A} \in \mathcal{O}_{b}$ and $\mathbf{A}_{n} \rightarrow \mathbf{A}$. To see these, let $\epsilon>0$. Then there exists a positive integer $N$ such that for each positive integer $K$,

$$
\left\|\sum_{k=1}^{K}\left(A_{k}^{(n)}-A_{k}^{(m)}\right)^{[2]}\right\|^{1 / 2} \leq\left\|\mathbf{A}_{n}-\mathbf{A}_{m}\right\|<\frac{\epsilon}{2} \text { for all } n, m \geq N .
$$


Since $A_{k}^{(m)} \rightarrow A_{k}$ in $\mathcal{S}^{2}$ for all $k$, it follows for each fixed $n$ that $A_{k}^{(n)}-A_{k}^{(m)} \rightarrow$ $A_{k}^{(n)}-A_{k}$ in $\mathcal{S}^{2}$ for all $k$. Thus, by Proposition 2.4 , we have for each fixed $n$ that $\left(A_{k}^{(m)}-A_{k}^{(n)}\right)^{[2]} \rightarrow\left(A_{k}^{(n)}-A_{k}\right)^{[2]}$ in $\mathcal{B}\left(l_{2}\right)$ for all $k$. Hence, for each fixed $n$ and $K, \sum_{k=1}^{K}\left(A_{k}^{(n)}-A_{k}^{(m)}\right)^{[2]} \rightarrow \sum_{k=1}^{K}\left(A_{k}^{(n)}-A_{k}\right)^{[2]}$ in $\mathcal{B}\left(l_{2}\right)$. Therefore, by taking the limits as $m \rightarrow \infty$ on both sides of $(*)$, we have, for each $n \geq N$, by the continuity of the operator norm on $\mathcal{B}\left(l_{2}\right)$ that

$$
\left\|\sum_{k=1}^{K}\left(A_{k}^{(n)}-A_{k}\right)^{[2]}\right\|^{1 / 2} \leq \frac{\epsilon}{2} \text { for all } K \geq 1 .
$$

Consequently,

$$
\left\|\mathbf{A}_{n}-\mathbf{A}\right\| \mid<\epsilon \text { for all } n \geq N .
$$

From this, we obtain that $\mathbf{A}_{N}-\mathbf{A}$ belongs to $\mathcal{O}_{b}$, which implies that $\mathbf{A}=$ $\mathbf{A}_{N}-\left(\mathbf{A}_{N}-\mathbf{A}\right)$ belongs to $\mathcal{O}_{b}$. It follows that $\mathbf{A}_{n} \rightarrow \mathbf{A}$, thus $\left(\mathcal{O}_{b},\|\cdot\| \|\right)$ is a Banach space.

To see that $\mathcal{O}_{c}$ is a Banach space, suppose that $\left\{\mathbf{A}_{n}=\left\{A_{k}^{(n)}\right\}_{k=1}^{\infty}\right\}_{n=1}^{\infty}$ is a sequence in $\mathcal{O}_{c}$ converging to a point $\mathbf{A}=\left\{A_{k}\right\}_{k=1}^{\infty}$ in $\mathcal{O}_{b}$. We will show that $\mathbf{A} \in \mathcal{O}_{c}$. To see this, let $\epsilon>0$ be given. Then there exists a positive integer $N$ such that $\left\|\mathbf{A}_{N}-\mathbf{A}\right\|<\frac{\sqrt{\epsilon}}{2}$. Since $\mathbf{A}_{N} \in \mathcal{O}_{c}$, there is a positive integer $K$ such that

$$
\left\|\sum_{k=\nu}^{\mu}\left(A_{k}^{(N)}\right)^{[2]}\right\|<\frac{\epsilon}{4} \text { for all } \mu>\nu>K .
$$

Thus, by Minkowski's inequality,

$$
\begin{aligned}
\left\|\sum_{k=\nu}^{\mu} A_{k}^{[2]}\right\| & =\left\|\sum_{k=\nu}^{\mu}\left(\left(A_{k}^{(N)}-A_{k}\right)+A_{k}^{(N)}\right)^{[2]}\right\| \\
& \leq\left(\left\|\sum_{k=\nu}^{\mu}\left(A_{k}^{(N)}-A_{k}\right)^{[2]}\right\|^{1 / 2}+\| \sum_{k=\nu}^{\mu}\left(A_{k}^{(N)}\right)^{\left.[2] \|^{1 / 2}\right)^{2}}\right. \\
& \leq\left(\left\|\mathbf{A}_{N}-\mathbf{A}\right\|+\left\|\sum_{k=\nu}^{\mu}\left(A_{k}^{(N)}\right)^{[2]}\right\|^{1 / 2}\right)^{2} \\
& <\left(\frac{\sqrt{\epsilon}}{2}+\frac{\sqrt{\epsilon}}{2}\right)^{2}=\epsilon \text { for all } \mu>\nu>K .
\end{aligned}
$$

Therefore, $\mathbf{A} \in \mathcal{O}_{c}$; it follows that $\mathcal{O}_{c}$ is a closed subspace of $\mathcal{O}_{b}$. Consequently, $\left(\mathcal{O}_{c},|\|\cdot\||\right)$ is a Banach space.

In the space $\mathcal{O}_{c}$, Cauchy-Schwarz's inequality and Minkowski's inequality can be extended by the continuity of the operator norm on $\mathcal{B}\left(l_{2}\right)$ to infinite sums as follows.

Theorem 2.10. For any $\left\{A_{k}\right\}_{k=1}^{\infty}$ and $\left\{B_{k}\right\}_{k=1}^{\infty}$ in $\mathcal{O}_{c}$, 
(1) $\left\|\sum_{k=1}^{\infty}\left|A_{k} \bullet B_{k}\right|\right\| \leq\left\|\sum_{k=1}^{\infty} A_{k}^{[2]}\right\|^{1 / 2}\left\|\sum_{k=1}^{\infty} B_{k}^{[2]}\right\|^{1 / 2} ;$
(2) $\left\|\sum_{k=1}^{\infty}\left(A_{k}+B_{k}\right)^{[2]}\right\|^{1 / 2} \leq\left\|\sum_{k=1}^{\infty} A_{k}^{[2]}\right\|^{1 / 2}+\left\|\sum_{k=1}^{\infty} B_{k}^{[2]}\right\|^{1 / 2}$.

On the classical sequence space $l_{2}$, we have a result closely related to its duality that a sequence $\mathbf{x}$ belongs to $l_{2}$ if and only if $\mathbf{x}$ "Schur multiplies" every $\mathbf{y}$ in $l_{2}$ into $l_{1}$. In Proposition 2.11 below, we obtain similar duality-type results for the sequence spaces $\mathcal{O}_{b}$ and $\mathcal{O}_{c}$. Notice that by Proposition 2.2, the assertions stated in this proposition make sense.

Proposition 2.11. Let $\left\{A_{k}\right\}_{k=1}^{\infty}$ be a sequence in $\mathcal{S}^{2}$.

(1) The sequence $\left\{A_{k}\right\}_{k=1}^{\infty}$ belongs to $\mathcal{O}_{c}$ if and only if the series $\sum_{k=1}^{\infty} \mid A_{k} \bullet$ $B_{k} \mid$ converges in $\mathcal{B}\left(l_{2}\right)$ for all $\left\{B_{k}\right\}_{k=1}^{\infty} \in \mathcal{O}_{b}$. If $\left\{A_{k}\right\}_{k=1}^{\infty}$ belongs to $\mathcal{O}_{c}$, then

$$
\left\|\left\{A_{k}\right\}_{k=1}^{\infty}\right\| \mid=\sup \left\{\left\|\sum_{k=1}^{\infty} A_{k} \bullet B_{k}\right\|:\left\{B_{k}\right\}_{k=1}^{\infty} \in \mathcal{O}_{b},\left\|\left\{B_{k}\right\}_{k=1}^{\infty}\right\| \leq 1\right\} .
$$

(2) The sequence $\left\{A_{k}\right\}_{k=1}^{\infty}$ belongs to $\mathcal{O}_{b}$ if and only if the series $\sum_{k=1}^{\infty} \mid A_{k} \bullet$ $B_{k} \mid$ converges in $\mathcal{B}\left(l_{2}\right)$ for all $\left\{B_{k}\right\}_{k=1}^{\infty} \in \mathcal{O}_{c}$. If $\left\{A_{k}\right\}_{k=1}^{\infty}$ belongs to $\mathcal{O}_{b}$, then

$$
\left\|\left\{A_{k}\right\}_{k=1}^{\infty}\right\| \mid=\sup \left\{\left\|\sum_{k=1}^{\infty} A_{k} \bullet B_{k}\right\|:\left\{B_{k}\right\}_{k=1}^{\infty} \in \mathcal{O}_{c}, \quad\left\|\left\{B_{k}\right\}_{k=1}^{\infty}\right\| \leq 1\right\} .
$$

Proof. (1) Suppose that the series $\sum_{k=1}^{\infty}\left|A_{k} \bullet B_{k}\right|$ converges in $\mathcal{B}\left(l_{2}\right)$ for all $\left\{B_{k}\right\}_{k=1}^{\infty} \in \mathcal{O}_{b}$. Then the linear operator $\Psi$ from $\mathcal{O}_{b}$ into $\mathcal{B}\left(l_{2}\right)$ defined by $\Psi\left(\left\{B_{k}\right\}_{k=1}^{\infty}\right)=\sum_{k=1}^{\infty} A_{k} \bullet B_{k}$ for all $\left\{B_{k}\right\}_{k=1}^{\infty} \in \mathcal{O}_{b}$ is well-defined. To see that $\Psi$ is bounded, let, for each $n, \Psi_{n}$ be the linear operator from $\mathcal{O}_{b}$ into $\mathcal{B}\left(l_{2}\right)$ defined by $\Psi_{n}\left(\left\{B_{k}\right\}_{k=1}^{\infty}\right)=\sum_{k=1}^{n} A_{k} \bullet B_{k}$ for all $\left\{B_{k}\right\}_{k=1}^{\infty} \in \mathcal{O}_{b}$. Then by Cauchy-Schwrz's inequality, $\Psi_{n}$ is bounded for all $n$. For each $\left\{B_{k}\right\}_{k=1}^{\infty}$ in $\mathcal{O}_{b}$, we have $\left\|\Psi_{n}\left(\left\{B_{k}\right\}_{k=1}^{\infty}\right)\right\| \leq\left\|\sum_{k=1}^{\infty}\left|A_{k} \bullet B_{k}\right|\right\|$ for all $n$. So, by the uniform boundedness principle, $\sup _{n}\left\|\Psi_{n}\right\|<\infty$. It follows that $\Psi$ is bounded. For each $n$, we have

$$
\begin{aligned}
\left\|\sum_{k=1}^{n} A_{k}^{[2]}\right\| & =\left\|\sum_{k=1}^{n} A_{k} \bullet \overline{A_{k}}\right\|=\left\|\Psi\left(\left\{\overline{A_{1}}, \overline{A_{2}}, \ldots, \overline{A_{n}}, 0,0, \ldots\right\}\right)\right\| \\
& \leq\|\Psi\| \mid\left\|\left\{\overline{A_{1}}, \overline{A_{2}}, \ldots, \overline{A_{n}}, 0,0, \ldots\right\}\right\|=\|\Psi\|\left\|\sum_{k=1}^{n} A_{k}^{[2]}\right\|^{1 / 2} .
\end{aligned}
$$

It follows that $\left\|\sum_{k=1}^{n} A_{k}^{[2]}\right\| \leq\|\Psi\|^{2}$ for all $n$, and hence $\left\{\overline{A_{k}}\right\}_{k=1}^{\infty} \in \mathcal{O}_{b}$. Thus, by the assumption, $\left\{\sum_{k=1}^{n} A_{k}^{[2]}\right\}_{n=1}^{\infty}=\left\{\sum_{k=1}^{n} A_{k} \bullet \overline{A_{k}}\right\}_{n=1}^{\infty}$ converges in $\mathcal{B}\left(l_{2}\right)$. 
Therefore $\left\{A_{k}\right\}_{k=1}^{\infty} \in \mathcal{O}_{c}$. The converse follows immediately from CauchySchwrz's inequality.

If $\left\{A_{k}\right\}_{k=1}^{\infty} \in \mathcal{O}_{c}$, then the operator $\Psi$ defined above is bounded, and we also have that $\left\|\left\{A_{k}\right\}_{k=1}^{\infty}\right\| \leq\|\Psi\|$. By Cauchy-Schwarz's inequality, we have for every $\left\{B_{k}\right\}_{k=1}^{\infty} \in \mathcal{O}_{b}$ with $\left\|\left\{B_{k}\right\}_{k=1}^{\infty}\right\| \mid \leq 1$ that

$$
\begin{aligned}
\left\|\Psi\left(\left\{B_{k}\right\}_{k=1}^{\infty}\right)\right\| & =\left\|\sum_{k=1}^{\infty} A_{k} \bullet B_{k}\right\|=\lim _{n \rightarrow \infty}\left\|\sum_{k=1}^{n} A_{k} \bullet B_{k}\right\| \\
& \leq \lim _{n \rightarrow \infty}\left\|\sum_{k=1}^{n} A_{k}^{[2]}\right\|^{1 / 2}\left\|\sum_{k=1}^{n} B_{k}^{[2]}\right\|^{1 / 2} \\
& \leq\left\|\left\{B_{k}\right\}_{k=1}^{\infty}\right\| \mid\left(\lim _{n \rightarrow \infty}\left\|\sum_{k=1}^{n} A_{k}^{[2]}\right\|^{1 / 2}\right) \\
& =\left\|\left\{B_{k}\right\}_{k=1}^{\infty}\right\|\left\|\sum_{k=1}^{\infty} A_{k}^{[2]}\right\|^{1 / 2} \leq\left\|\left\{A_{k}\right\}_{k=1}^{\infty}\right\| .
\end{aligned}
$$

It follows that $\|\Psi\| \leq\left|\left\|\left\{A_{k}\right\}_{k=1}^{\infty}\right\|\right|$.

(2) The proof is similar to that of (1), so omitted.

Another space of sequences in $\mathcal{S}^{2}$ which can reasonably be defined is the following:

$$
\mathcal{O}_{k}:=\left\{\left\{A_{k}=\left[a_{j i}^{(k)}\right]\right\}_{k=1}^{\infty} \subset \mathcal{S}^{2}: \text { the matrix }\left[\sum_{k=1}^{\infty}\left|a_{j i}^{(k)}\right|^{2}\right] \text { is compact }\right\} .
$$

For any matrix $A$ and positive integer $n$, let $A_{\lrcorner_{n}}:=A-A_{n}$. It is apparent that a sequence $\left\{A_{k}\right\}_{k}^{\infty}$ belongs to $\mathcal{O}_{k}$ if and only if $\left\|\left\{\left(A_{k}\right)_{n}\right\}_{k=1}^{\infty}\right\| \mid \rightarrow 0$. Thus, by the continuity of the map $A \mapsto A^{[2]}$ from $\mathcal{S}^{2}$ into $\mathcal{B}\left(l_{2}\right)$, we obtain that if $\left\{A_{k}\right\}_{k=1}^{\infty} \in \mathcal{O}_{k}$, then $A_{k}^{[2]}$ is necessarily compact for all $k$.

From Proposition 2.6(2), we have that $\mathcal{O}_{k} \subseteq \mathcal{O}_{c}$. The containment is obviously proper, indeed, any sequence having the first term a matrix $A$ whose $A^{[2]}$ is noncompact (such as the identity matrix) and other terms 0 , belongs to $\mathcal{O}_{c}$ but doesn't belong to $\mathcal{O}_{k}$. Notice that there is an unbounded matrix $A$ such that $A^{[2]}$ is compact, for example, the matrix $A$ whose first column is the sequence $\left\{\frac{1}{\sqrt{k}}\right\}_{k=1}^{\infty}$ and all other columns are 0 . From this result, $\mathcal{O}_{k}$ contains some sequences of unbounded matrices, for instance, the sequence $\left\{A_{k}\right\}_{k=1}^{\infty}$ such that $A_{k}=\sqrt{\frac{1}{2^{k}}} A$, where $A$ is the matrix defined above, which clearly belongs to $\mathcal{O}_{k}$.

Theorem 2.12. A sequence $\mathbf{A}=\left\{A_{k}\right\}_{k=1}^{\infty}$ belongs to $\mathcal{O}_{k}$ if and only if $A_{k}^{[2]}$ is compact for all $k$ and $\mathbf{A}$ belongs to $\mathcal{O}_{c}$. 
Proof. Suppose that $A_{k}^{[2]}$ is compact for all $k$ and that $\mathbf{A}$ belongs to $\mathcal{O}_{c}$. Then $\sum_{k=1}^{n} A_{k}^{[2]}$ is compact for all $n$. Since $\left\{\sum_{k=1}^{n} A_{k}^{[2]}\right\}_{n=1}^{\infty}$ converges in $\mathcal{B}\left(l_{2}\right)$, we have by the closedness of the space of compact operators in $\mathcal{B}\left(l_{2}\right)$ that the matrix $\sum_{k=1}^{\infty} A_{k}^{[2]}$ is compact. The converse was already obtained from the discussion above.

Theorem 2.13. The set $\mathcal{O}_{k}$ is a Banach space under the norm $\|\cdot\| \mid$.

Proof. For any $\left\{A_{k}\right\}_{k=1}^{\infty},\left\{B_{k}\right\}_{k=1}^{\infty} \in \mathcal{O}_{k}$, we have

$$
\begin{aligned}
\left\|\left\{\left(A_{k}+B_{k}\right)_{\lrcorner_{n}}\right\}_{k=1}^{\infty}\right\| \mid & =\left|\left\|\left\{\left(A_{k}\right)_{\lrcorner_{n}}\right\}_{k=1}^{\infty}+\left\{\left(B_{k}\right)_{n}\right\}_{k=1}^{\infty}\right\|\right| \\
& \leq\left|\left\|\left\{\left(A_{k}\right)_{\lrcorner_{n}}\right\}_{k=1}^{\infty}\right\|\right|+\left|\left\|\left\{\left(B_{k}\right)_{\lrcorner_{n}}\right\}_{k=1}^{\infty}\right\|\right| \rightarrow 0 .
\end{aligned}
$$

Thus $\mathcal{O}_{k}$ is closed under addition. It follows that $\mathcal{O}_{k}$ is a normed space under the norm $\|\cdot\| \|$. Suppose that $\left\{\mathbf{A}_{n}=\left\{A_{k}^{(n)}\right\}_{k=1}^{\infty}\right\}_{n=1}^{\infty}$ is a sequence in $\mathcal{O}_{k}$ converging to an element $\mathbf{A}=\left\{A_{k}\right\}_{k=1}^{\infty}$ in $\mathcal{O}_{c}$. Let $\epsilon>0$. Then there is a positive integer $N$ such that $\left\|\mathbf{A}_{N}-\mathbf{A}\right\| \mid<\frac{\epsilon}{2}$. Since $\mathbf{A}_{N} \in \mathcal{O}_{k}$, there exists a positive integer $J_{0}$ such that

$$
\left\|\left\{\left(A_{k}^{(N)}\right)_{\lrcorner_{J}}\right\}_{k=1}^{\infty}\right\| \mid<\frac{\epsilon}{2} \text { for all } J \geq J_{0} .
$$

It follows that

$$
\begin{aligned}
& \left\|\left\{\left(A_{k}\right)_{\lrcorner}\right\}_{k=1}^{\infty}\right\||\leq|\left\|\left\{\left(A_{k}^{(N)}\right)_{\lrcorner_{J}}\right\}_{k=1}^{\infty}-\left\{\left(A_{k}\right)_{\lrcorner}\right\}_{k=1}^{\infty}\right\|\|+\|\left\{\left(A_{k}^{(N)}\right)_{\lrcorner_{J}}\right\}_{k=1}^{\infty} \| \\
& =\left\|\left\{\left(A_{k}^{(N)}-A_{k}\right)_{\lrcorner J}\right\}_{k=1}^{\infty}\right\|\|+\|\left\{\left(A_{k}^{(N)}\right)_{\lrcorner_{J}}\right\}_{k=1}^{\infty} \| \\
& \leq\left\|\mathbf{A}_{N}-\mathbf{A}\right\|+\left\|\left\{\left(A_{k}^{(N)}\right)_{\lrcorner_{J}}\right\}_{k=1}^{\infty}\right\| \| \\
& <\frac{\epsilon}{2}+\frac{\epsilon}{2}=\epsilon \text { for all } J \geq J_{0} .
\end{aligned}
$$

Consequently, $\sum_{k=1}^{\infty} A_{k}^{[2]}$ is compact.

Remark 2.14. We note here the following observations.

(1) By Schur-Bennett's theorem, $\mathcal{B}\left(l_{2}\right)$ equipped with the Schur product operation and the operator norm is a Banach algebra. From this result, the three sequence spaces $\mathcal{O}_{b}, \mathcal{O}_{c}$, and $\mathcal{O}_{k}$ can be defined on the Banach algebra $\mathcal{B}\left(l_{2}\right)$ as well, but they are not complete. In fact, we can choose by the non-closedness of $\mathcal{B}\left(l_{2}\right)$ in $\mathcal{S}^{2}$ a sequence of finite-rank matrices in $\mathcal{B}\left(l_{2}\right)$ that converges to an unbounded matrix in $\mathcal{S}^{2}$. Such a sequence induces a Cauchy sequence in each of the three sequence spaces which is not convergent.

(2) Another sequence space of operators on $l_{2}$ which might raise some interesting questions is the space $\mathcal{A}$ of sequences $\left\{A_{k}\right\}_{k=1}^{\infty}$ of absolutely bounded operators whose $\sum_{k=1}^{\infty}\left|A_{k}\right|$ converges. It is not hard to show 
that $\mathcal{A}$ is a Banach space under the norm defined to be the operator norm of the corresponding matrix sum.

\section{Sequential convergence}

In this section, we study sequential convergence in our sequence spaces of matrices. We expect to have a characterization of convergent sequences in the sequence spaces of matrices in terms of some sequential convergence modes of corresponding matrix entries. In [3], I. E. Leonard gave a characterization of norm convergence of sequences in the classical Banach sequence spaces $l_{p}(X)$ in terms of norm convergence of sequences in the Banach space $X$. A similar result is also obtained in the sequence space $\mathcal{O}_{c}$.

For any sequence $\mathbf{A}=\left\{A_{k}\right\}_{k=1}^{\infty}$ of matrices and any positive integer $K$, we let here $\mathbf{A}_{[K}:=\left\{0,0, \ldots, 0, A_{K}, A_{K+1}, \ldots\right\}$.

Theorem 3.1. Let $\left\{\mathbf{A}_{n}=\left\{A_{k}^{(n)}\right\}_{k=1}^{\infty}\right\}_{n=1}^{\infty}$ be a sequence in $\mathcal{O}_{c}$ and $\mathbf{A}=$ $\left\{A_{k}\right\}_{k=1}^{\infty} \in \mathcal{O}_{b}$. Then $\mathbf{A}_{n} \rightarrow \mathbf{A}$ if and only if

(1) $\mathbf{A} \in \mathcal{O}_{c}$;

(2) $A_{k}^{(n)} \rightarrow A_{k}$ in $\mathcal{S}^{2}$ for all $k$;

(3) $\left\|\left(\mathbf{A}_{n}\right)_{[K}\right\||\rightarrow|\left\|\mathbf{A}_{[K}\right\| \mid$ for all $K$.

Proof. Suppose that $\left\{\mathbf{A}_{n}=\left\{A_{k}^{(n)}\right\}_{k=1}^{\infty}\right\}_{n=1}^{\infty}$ converges to $\mathbf{A}=\left\{A_{k}\right\}_{k=1}^{\infty}$. Then by the closedness of $\mathcal{O}_{c}$ in $\mathcal{O}_{b}$, we have $\mathbf{A} \in \mathcal{O}_{c}$. It is evident that $A_{k}^{(n)} \rightarrow A_{k}$ for all $k$. Since $\mathbf{A}_{n} \rightarrow \mathbf{A}$, it is easy to see that $\left(\mathbf{A}_{n}\right)_{[K} \rightarrow A_{[K}$ for all $K$. Thus, by the continuity of the norm $\|\cdot\| \mid \|$, we have $\left\|\left(\mathbf{A}_{n}\right)_{[K}\right\|\|\rightarrow\| \mathbf{A}_{[K} \| \mid$ for all $K$. Conversely, suppose that the conditions (1)-(3) hold. For each integer $K \geq 1$, we have by Minkowski's inequality that

$$
\begin{aligned}
& \left\|\sum_{k=1}^{\infty}\left(A_{k}^{(n)}-A_{k}\right)^{[2]}\right\| \\
\leq & \left\|\sum_{k=1}^{K}\left(A_{k}^{(n)}-A_{k}\right)^{[2]}\right\|+\left\|\sum_{k=K+1}^{\infty}\left(A_{k}^{(n)}-A_{k}\right)^{[2]}\right\| \\
\leq & \sum_{k=1}^{K}\left\|\left(A_{k}^{(n)}-A_{k}\right)^{[2]}\right\|+\left\{\left\|\sum_{k=K+1}^{\infty}\left(A_{k}^{(n)}\right)^{[2]}\right\|^{1 / 2}+\left\|\sum_{k=K+1}^{\infty} A_{k}^{[2]}\right\|^{1 / 2}\right\}^{2} \text { for all } n .
\end{aligned}
$$

By taking the limit as $n \rightarrow \infty$, we have

$$
\limsup _{n \rightarrow \infty}\left\|\mathbf{A}_{n}-\mathbf{A}\right\|=\limsup _{n \rightarrow \infty}\left\|\sum_{k=1}^{\infty}\left(A_{k}^{(n)}-A_{k}\right)^{[2]}\right\|^{1 / 2} \leq 2\left\|\sum_{k=K+1}^{\infty} A_{k}^{[2]}\right\|^{1 / 2} .
$$

Hence, by taking the limit as $K \rightarrow \infty$, we obtain $\lim \sup _{n \rightarrow \infty}\left\|\mathbf{A}_{n}-\mathbf{A}\right\|=0$. This yields $\lim _{n \rightarrow \infty}\left|\left\|\mathbf{A}_{n}-\mathbf{A}\right\|\right|=0$. The proof is complete. 
We obtain as expected a further characterization of norm convergence of sequences in the sequence space $\mathcal{O}_{k}$ in terms of norm convergence, in the space $l_{2}$, of sequences of the matrix rows from the corresponding sequence of matrix sums.

We denote here, for any sequence $\mathbf{A}=\left\{A_{k}\right\}_{k=1}^{\infty}$ of matrices and any positive integer $J$, the sequence $\left\{A_{k}-\left(A_{k}\right)_{\underline{J}}\right\}_{k=1}^{\infty}$ by $\mathbf{A}_{\bar{J}}$.

Theorem 3.2. Let $\left\{\mathbf{A}_{n}=\left\{\left[a_{j i}^{(k, n)}\right]\right\}_{k=1}^{\infty}\right\}_{n=1}^{\infty}$ be a sequence in the space $\mathcal{O}_{k}$, and let $\mathbf{A}=\left\{\left[a_{j i}^{(k)}\right]\right\}_{k=1}^{\infty}$ be an element in $\mathcal{O}_{b}$. Then $\mathbf{A}_{n} \rightarrow \mathbf{A}$ if and only if

(1) $\mathbf{A} \in \mathcal{O}_{k}$;

(2) $\left\{\sum_{k=1}^{\infty}\left|a_{j i}^{(k, n)}-a_{j i}^{(k)}\right|^{2}\right\}_{i=1}^{\infty} \rightarrow 0$ in $l_{2}$ for all $j$;

(3) $\left\|\left(\mathbf{A}_{n}\right)_{\bar{J}}\right\| \rightarrow\left\|\mathbf{A}_{\bar{J}}\right\|$ for all $J$.

Proof. Suppose that $\mathbf{A}_{n} \rightarrow \mathbf{A}$. Then by the closedness of $\mathcal{O}_{k}$ in $\mathcal{O}_{b}$, the condition (1) holds. From the well-known fact that if $A$ belongs to $\mathcal{B}\left(l_{2}\right)$, then each row of $A$ belongs to $l_{2}$ and has the $l_{2}$-norm less than or equal to the operator norm of $A$, we have the condition (2) is satisfied. Since $\mathbf{A}_{n} \rightarrow \mathbf{A}$, it is evident that $\left(\mathbf{A}_{n}\right)_{\bar{J}} \rightarrow \mathbf{A}_{\bar{J}}$ for all $J$. Thus, by continuity of the norm $\|\cdot\| \mid,(3)$ is obtained. Conversely, suppose that the conditions (1)-(3) are satisfied. Notice that for any $a, b, r>0,(a+b)^{r} \leq 2^{r}\left(a^{r}+b^{r}\right)$. Then for each fixed $J$, we have for any $\mathbf{x}=\left\{x_{i}\right\}_{i=1}^{\infty} \in l_{2}$ with $\|\mathbf{x}\|_{2} \leq 1$ that

$$
\begin{aligned}
\left\|\left[\sum_{k=1}^{\infty}\left|a_{j i}^{(k, n)}-a_{j i}^{(k)}\right|^{2}\right] \mathbf{x}\right\|_{2}^{2} \leq & \sum_{j=1}^{\infty}\left(\sum_{i=1}^{\infty} \sum_{k=1}^{\infty}\left|a_{j i}^{(k, n)}-a_{j i}^{(k)}\right|^{2}\left|x_{i}\right|\right)^{2} \\
= & \sum_{j=J+1}^{\infty}\left(\sum_{i=1}^{\infty} \sum_{k=1}^{\infty}\left|a_{j i}^{(k, n)}-a_{j i}^{(k)}\right|^{2}\left|x_{i}\right|\right)^{2} \\
& +\sum_{j=1}^{j=J}\left(\sum_{i=1}^{\infty} \sum_{k=1}^{\infty}\left|a_{j i}^{(k, n)}-a_{j i}^{(k)}\right|^{2}\left|x_{i}\right|\right)^{2} \\
\leq & 64\left(\left|\left\|\left(\mathbf{A}_{n}\right)_{\bar{J}}\right\|\right|^{4}+|| \mathbf{A}_{\bar{J}} \|\left.\right|^{4}\right) \\
& +\sum_{j=1}^{\infty} \sum_{i=1}^{\infty}\left(\sum_{k=1}^{\infty}\left|a_{j i}^{(k, n)}-a_{j i}^{(k)}\right|^{2}\right)^{2} \text { for all } n .
\end{aligned}
$$

Thus, for each $J$, we obtain for every $n$ that

$$
\left\|\mathbf{A}_{n}-\mathbf{A}\right\| \leq\left\{64\left(\left\|\left(\mathbf{A}_{n}\right)_{\bar{J}}\right\|^{4}+\left\|\mathbf{A}_{\bar{J}}\right\|^{4}\right)+\sum_{j=1}^{j=J} \sum_{i=1}^{\infty}\left(\sum_{k=1}^{\infty}\left|a_{j i}^{(k, n)}-a_{j i}^{(k)}\right|^{2}\right)^{2}\right\}^{1 / 4} .
$$


Hence, for each $J$,

$$
\limsup _{n \rightarrow \infty}\left\|\mathbf{A}_{n}-\mathbf{A}\right\| \leq \sqrt[4]{128}\left\|\mathbf{A}_{\bar{J}}\right\|=\sqrt[4]{128}\left\|\left(\sum_{k=1}^{\infty} A_{k}^{[2]}\right)_{\underline{J}}-\sum_{k=1}^{\infty} A_{k}^{[2]}\right\|^{1 / 2} .
$$

Therefore, by the compactness of the matrix $\sum_{k=1}^{\infty} A_{k}^{[2]}$, we have by taking the limit as $J \rightarrow \infty$ that $\lim \sup _{n \rightarrow \infty}\left\|\mathbf{A}_{n}-\mathbf{A}\right\|=0$. It follows that $\lim _{n \rightarrow \infty}\left\|\mathbf{A}_{n}-\mathbf{A}\right\|=0$. Consequently, we obtain $\mathbf{A}_{n} \rightarrow \mathbf{A}$ as asserted. The proof is finished.

\section{Duality and preduality of $\mathcal{O}_{k}$}

In this section we study the duality and preduality of the sequence space $\mathcal{O}_{k}$.

Let $\mathcal{K}^{2}$ be the set of all infinite matrices $A$ whose $A^{[2]}$ is compact as an operator on $l_{2}$. It was proved in [4] that $\mathcal{K}^{2}$ is the closure in $\mathcal{S}^{2}$ of the set of all matrices with finitely many non-zero entries, and moreover $A \in \mathcal{K}^{2}$ if and only if $\left\|A_{n_{\lrcorner}}-A\right\|_{2} \rightarrow 0$. By Theorem 2.12, we see that for a sequence $\left\{A_{k}=\left[a_{j i}^{(k)}\right]\right\}_{k=1}^{\infty}$ in $\mathcal{K}^{2}$, the matrix $\left[\sum_{k=1}^{\infty}\left|a_{j i}^{(k)}\right|^{2}\right]$ is compact if and only if the series $\sum_{k=1}^{\infty} A_{k}^{[2]}$ converges.

For any $z \in \mathbb{C}$, let $\operatorname{sgn}(z)=\frac{\bar{z}}{|z|}$ if $z \neq 0$ and $\operatorname{sgn}(z)=1$ otherwise.

Theorem 4.1. The space $\mathcal{O}_{k}$ cannot be the dual space of any normed space.

Proof. We will show first that the closed unit ball of $\mathcal{O}_{k}$ has no extreme points. Let $\mathbf{A}=\left\{A_{k}\right\}_{k=1}^{\infty} \in \mathcal{O}_{k}$ with $\|\mathbf{A}\|=1$. Then there is $n_{0}$ such that

$$
\left\|\sum_{k=1}^{\infty} A_{k}^{[2]}-\left(\sum_{k=1}^{\infty} A_{k}^{[2]}\right)_{\underline{n_{0}}}\right\|<\frac{1}{2} .
$$

Let, for each $k, E_{k}$ be the matrix whose $\left(n_{0}+1, k\right)$-th entry is $\frac{1}{\sqrt{2}}\left(\frac{1}{2}\right)^{\frac{k}{4}}$ and all other entries are 0 , and let $\mathbf{B}=\left\{A_{k}+E_{k}\right\}_{k=1}^{\infty}$ and $\mathbf{C}=\left\{A_{k}-E_{k}\right\}_{k=1}^{\infty}$. It is clear that $\left\{E_{k}\right\}_{k=1}^{\infty}$ belongs to $\mathcal{O}_{k}$ (in fact, $\sum_{k=1}^{\infty} E_{k}^{[2]}$ is a rank-1 operator with the norm $\frac{1}{2}$ ). Thus $\mathbf{B}$ and $\mathbf{C}$ belong to $\mathcal{O}_{k}$ and $\mathbf{A}=\frac{1}{2} \mathbf{B}+\frac{1}{2} \mathbf{C}$. From $(* *)$ and our Minkowski's inequality for sequences of matrices, we obtain

$$
\|\mathbf{B}\|=\max \left\{\left\|\left(\sum_{k=1}^{\infty} A_{k}^{[2]}\right)_{\underline{n_{0}}}\right\|,\left\|\sum_{k=1}^{\infty}\left(A_{k}+E_{k}\right)^{[2]}-\left(\sum_{k=1}^{\infty}\left(A_{k}+E_{k}\right)^{[2]}\right)_{\underline{n_{0}}}\right\|\right\}^{1 / 2} \leq 1,
$$

and

$$
\|\mathbf{C}\|=\max \left\{\left\|\left(\sum_{k=1}^{\infty} A_{k}^{[2]}\right)_{\underline{n_{0}}}\right\|,\left\|\sum_{k=1}^{\infty}\left(A_{k}-E_{k}\right)^{[2]}-\left(\sum_{k=1}^{\infty}\left(A_{k}-E_{k}\right)^{[2]}\right)_{\underline{n_{0}}}\right\|\right\}^{1 / 2} \leq 1 .
$$


Consequently, $\mathrm{A}$ is not an extreme point of the closed unit ball of $\mathcal{O}_{k}$. It follows that the closed unit ball of $\mathcal{O}_{k}$ has no extreme points. If $\mathcal{O}_{k}$ were the dual of a normed space, then by the Banach-Alaoglu theorem and the Krein-Milman theorem, the closed unit ball of $\mathcal{O}_{k}$ would have to contain at least one extreme point. This contradicts to the fact above. The proof is complete.

We next investigate the duality of $\mathcal{O}_{k}$. For any matrix $A=\left[a_{j i}\right]$, let $\sum A=$ $\sum_{j=1}^{\infty} \sum_{i=1}^{\infty} a_{j i}$ if the sum $\sum_{j=1}^{\infty} \sum_{i=1}^{\infty} a_{j i}$ converges. Let

$$
\mathcal{M}=\left\{B: \sum|A \bullet B|<\infty \text { for all } A \in \mathcal{K}^{2}\right\} .
$$

By the closed graph theorem, the norm

$$
\|B\|_{\mathcal{M}}=\sup \left\{\sum|A \bullet B|: A \in \mathcal{K}^{2}, \mid\|A\|_{2} \leq 1\right\}
$$

is a well-defined norm on $\mathcal{M}$.

Lemma 4.2. The set $\mathcal{M}$ equipped with the norm $\|\cdot\|_{\mathcal{M}}$ is a Banach space.

Proof. Let $\left\{B_{n}=\left[b_{j i}^{(n)}\right]\right\}_{n=1}^{\infty}$ be a Cauchy sequence in $\mathcal{M}$. Notice that for any $\left[c_{j i}\right]$ in $\mathcal{M},\left|c_{j i}\right| \leq\left\|\left[c_{j i}\right]\right\|_{\mathcal{M}}$. Then for each $(j, i)$, the sequence $\left\{b_{j i}^{(n)}\right\}_{n=1}^{\infty}$ is a Cauchy sequence in $\mathbb{C}$. Let $b_{j i}=\lim _{n \rightarrow \infty} b_{j i}^{(n)}$, and put $B=\left[b_{j k}\right]$. We will prove that $B \in \mathcal{M}$ and $B_{n} \rightarrow B$. Let $\epsilon>0$. Then there is a positive integer $N$ such that for any $A \in \mathcal{K}^{2}$ with $\|A\|_{2} \leq 1$ and any $J \geq 1$,

$$
\sum\left|A_{J_{\lrcorner}} \bullet\left(B_{n}-B_{m}\right)\right| \leq\left\|B_{n}-B_{m}\right\|_{\mathcal{M}}<\frac{\epsilon}{2} \text { for all } n, m \geq N .
$$

Thus, by taking the limit as $m \rightarrow \infty$,

$$
\sum\left|A_{J_{\lrcorner}} \bullet\left(B_{n}-B\right)\right| \leq \frac{\epsilon}{2} \text { for all } n \geq N .
$$

It follows for any $A \in \mathcal{K}^{2}$ with $\|A\|_{2} \leq 1$ that

$$
\sum\left|A \bullet\left(B_{n}-B\right)\right| \leq \frac{\epsilon}{2} \text { for all } n \geq N .
$$

This implies that $B_{N}-B \in \mathcal{M}$. Thus $B \in \mathcal{M}$, and hence $B_{n} \rightarrow B$.

Let

$$
\triangle=\left\{\left\{B_{k}\right\}_{k=1}^{\infty} \subset \mathcal{M}: \sum_{k=1}^{\infty}\left|\sum A_{k} \bullet B_{k}\right|<\infty \text { for all }\left\{A_{k}\right\}_{k=1}^{\infty} \in \mathcal{O}_{k}\right\} .
$$

It can also be shown by the closed graph theorem that

$\left\|\left\{B_{k}\right\}_{k=1}^{\infty}\right\|_{*}:=\sup \left\{\sum_{k=1}^{\infty}\left|\sum A_{k} \bullet B_{k}\right|:\left\{A_{k}\right\}_{k=1}^{\infty} \in \mathcal{O}_{k},\left\|\left\{A_{k}\right\}_{k=1}^{\infty}\right\| \mid \leq 1\right\}<\infty$

for all $\left\{B_{k}\right\}_{k=1}^{\infty} \in \triangle$.

Lemma 4.3. For any $\mathbf{B}=\left\{B_{k}\right\}_{k=1}^{\infty}$ in $\triangle$, $\left\|B_{k}\right\|_{\mathcal{M}} \leq\|\mathbf{B}\|_{*}$. 
Proof. For any $A$ in $\mathcal{K}^{2}$ with $\|A\|_{2} \leq 1$, let $\widetilde{A}_{k}=\left(\operatorname{sgn} \sum A \bullet B_{k}\right) A$ for all $k$, and let $C_{k}=\widetilde{A}_{k}$ and $C_{i}=0$ otherwise. Then $\left\{C_{i}\right\}_{i=1}^{\infty} \in \mathcal{O}_{k}$ and $\left\|\left\{C_{i}\right\}_{i=1}^{\infty}\right\| \mid=$ $\mid\|A\|_{2} \leq 1$. Thus $\sum\left|A \bullet B_{k}\right|=\sum_{i=1}^{\infty} \sum C_{i} \bullet B_{i} \leq\|\mathbf{B}\|_{*}$. Accordingly, $\left\|B_{k}\right\|_{\mathcal{M}} \leq$ $\|\mathbf{B}\|_{*}$.

Theorem 4.4. The set $\triangle$ equipped with the norm $\|\cdot\|_{*}$ is a Banach space.

Proof. Let $\left\{\mathbf{B}_{n}=\left\{B_{k}^{(n)}\right\}_{k=1}^{\infty}\right\}_{n=1}^{\infty}$ be a Cauchy sequence in $\triangle$. By Lemma 4.3, we have for any $k$ that

$$
\left\|B_{k}^{(n)}-B_{k}^{(m)}\right\|_{\mathcal{M}} \leq\left\|\mathbf{B}_{n}-\mathbf{B}_{m}\right\|_{*} \text { for all } m, n .
$$

It follows that $\left\{B_{k}^{(n)}\right\}_{n=1}^{\infty}$ is a Cauchy sequence in $\mathcal{M}$ for all $k$. Thus, by the completeness of $\mathcal{M}$, there is for each $k$ a matrix $B_{k}$ in $\mathcal{M}$ such that $B_{k}^{(n)} \rightarrow B_{k}$. Let $\mathbf{B}=\left\{B_{k}\right\}_{k=1}^{\infty}$. We claim that $\mathbf{B} \in \triangle$ and $\mathbf{B}_{n} \rightarrow \mathbf{B}$. To prove this, let $\epsilon>0$. Then there is a positive integer $N$ such that for any $\mathbf{A}=\left\{A_{k}\right\}_{k=1}^{\infty} \in \mathcal{O}_{k}$ with $\|\mathbf{A}\| \leq 1$,

$$
\sum_{k=1}^{\infty}\left|\sum A_{k} \bullet\left(B_{k}^{(n)}-B_{k}^{(m)}\right)\right| \leq\left\|\mathbf{B}_{n}-\mathbf{B}_{m}\right\|_{*}<\frac{\epsilon}{2} \text { for all } n, m \geq N .
$$

Since for each $k, B_{k}^{(m)} \rightarrow B_{k}$, we have $\sum A \bullet\left(B_{k}^{(m)}-B_{k}\right) \rightarrow 0$ for all $A \in \mathcal{K}^{2}$ and all $k$. Thus, by taking the limit as $m \rightarrow \infty$, we have for each $\mathbf{A}=$ $\left\{A_{k}\right\}_{k=1}^{\infty} \in \mathcal{O}_{k}$ with $\|\mathbf{A}\| \mid \leq 1$ that

$$
\sum_{k=1}^{\infty}\left|\sum A_{k} \bullet\left(B_{k}^{(n)}-B_{k}\right)\right| \leq \frac{\epsilon}{2} \text { for all } n \geq N .
$$

This implies that $\mathbf{B}_{N}-\mathbf{B} \in \triangle$. Thus $\mathbf{B} \in \triangle$, and therefore, $\mathbf{B}_{n} \rightarrow \mathbf{B}$.

The following duality theorem for $\mathcal{O}_{k}$ is obtained.

Theorem 4.5. $\mathcal{O}_{k}^{*}$ is isometrically isomorphic to $\triangle$.

Proof. Let $\mathbf{B}=\left\{B_{k}\right\}_{k=1}^{\infty} \in \triangle$. We then define a linear functional $\Psi_{\mathbf{B}}$ on $\mathcal{O}_{k}$ by $\Psi_{\mathbf{B}}(\mathbf{A})=\sum_{k=1}^{\infty} \sum A_{k} \bullet B_{k}$ for all $\mathbf{A}=\left\{A_{k}\right\}_{k=1}^{\infty}$ in $\mathcal{O}_{k}$. It is clear that $\Psi_{\mathbf{B}}$ is bounded and $\left\|\Psi_{\mathbf{B}}\right\| \leq\|\mathbf{B}\|_{*}$. To see that $\left\|\Psi_{\mathbf{B}}\right\| \geq\|\mathbf{B}\|_{*}$, let $\mathbf{A}=\left\{A_{k}\right\}_{k=1}^{\infty} \in$ $\mathcal{O}_{k}$ with $\|\mathbf{A}\| \leq 1$. Put $\widetilde{\mathbf{A}}=\left\{\left(\operatorname{sgn} \sum A_{k} \bullet B_{k}\right) A_{k}\right\}_{k=1}^{\infty}$. Then $\widetilde{\mathbf{A}} \in \mathcal{O}_{k}$ and $\|\widetilde{\mathbf{A}}\|=|\|\mathbf{A}\||$, and we have

$$
\begin{aligned}
\sum_{k=1}^{\infty}\left|\sum A_{k} \bullet B_{k}\right| & =\sum_{k=1}^{\infty} \sum\left(\operatorname{sgn} \sum A_{k} \bullet B_{k}\right) A_{k} \bullet B_{k} \\
& =\Psi_{\mathbf{B}}(\tilde{\mathbf{A}}) \leq\left\|\Psi_{\mathbf{B}}\right\| .
\end{aligned}
$$

Thus $\|\mathbf{B}\|_{*} \leq\left\|\Psi_{\mathbf{B}}\right\|$ as required. Next, let $\Phi \in \mathcal{O}_{k}^{*}$. For each $k$, let $\Phi^{(k)}$ be a linear functional on $\mathcal{K}^{2}$ defined by $\Phi^{(k)}(A)=\Phi(S(k ; A))$, where $S(k ; A)$ is the 
sequence whose $k$-th term is $A$ and all other terms 0 . Then $\Phi^{(k)} \in\left(\mathcal{K}^{2}\right)^{*}$ for all $k$. For each $k$, let $B_{k}=\left[\Phi^{(k)}(E(j, i))\right]$, where $E(j, i)$ is the matrix with the $(j, i)$-th entry 1 and all other entries 0 . Let $\mathbf{B}=\left\{B_{k}\right\}_{k=1}^{\infty}$. We are now going to show that $\mathbf{B} \in \triangle$. To see this, we need to show first that $B_{k} \in \mathcal{M}$ for all $k$. Let $A=\left[a_{j i}\right] \in \mathcal{K}^{2}$, and put $\widetilde{A}=\left[\operatorname{sgn}\left(a_{j i} \Phi^{(k)}(E(j, i))\right) a_{j i}\right]$. Then $\widetilde{A} \in \mathcal{K}^{2}$, and we have for each $k$ that

$$
\begin{aligned}
\sum\left|A_{n_{\lrcorner}} \bullet B_{k}\right| & =\sum \widetilde{A}_{n_{\lrcorner}} \bullet B_{k}=\Phi^{(k)}\left(\widetilde{A}_{n_{\lrcorner}}\right) \\
& \leq\left\|\Phi^{(k)}\right\|\|A\|_{2} \text { for all } n .
\end{aligned}
$$

It follows that $B_{k} \in \mathcal{M}$ for all $k$. Notice that for any $A \in \mathcal{K}^{2}$, we have for each $k$ that

$$
\Phi^{(k)}(A)=\lim _{n \rightarrow \infty} \Phi^{(k)}\left(A_{n\lrcorner}\right)=\lim _{n \rightarrow \infty} \sum_{j=1}^{n} \sum_{i=1}^{n} a_{j i} \Phi^{(k)}(E(j, i))=\sum A \bullet B_{k} .
$$

This give us for any $\mathbf{A}=\left\{A_{k}\right\}_{k=1}^{\infty}$ in $\mathcal{O}_{k}$ that

$$
\sum_{k=1}^{\infty} \sum A_{k} \bullet B_{k}=\sum_{k=1}^{\infty} \Phi^{(k)}\left(A_{k}\right)=\Phi(\mathbf{A})
$$

To show that $\mathbf{B} \in \triangle$, let $\mathbf{A}=\left\{A_{k}\right\}_{k=1}^{\infty} \in \mathcal{O}_{k}$, and define $\widetilde{\mathbf{A}}=\left\{\widetilde{A_{k}}\right\}_{k=1}^{\infty}$, where $\widetilde{A_{k}}$ is defined similarly as above. Then $\widetilde{\mathbf{A}}$ belongs to $\mathcal{O}_{k}$ and by the note above and its consequence, we obtain

$$
\begin{aligned}
\sum_{k=1}^{\infty}\left|\sum A_{k} \bullet B_{k}\right| & \leq \sum_{k=1}^{\infty} \sum\left|A_{k} \bullet B_{k}\right|=\sum_{k=1}^{\infty} \sum \widetilde{A_{k}} \bullet B_{k} \\
& =\sum_{k=1}^{\infty} \Phi^{(k)}\left(\widetilde{A_{k}}\right)=\Phi(\widetilde{\mathbf{A}}) .
\end{aligned}
$$

Hence $\mathbf{B} \in \triangle$, and thus $\Psi_{\mathbf{B}}=\Phi$. The proof is finished.

We end this paper with the reflexivity of the three sequence spaces.

Theorem 4.6. All the three sequence spaces $\mathcal{O}_{k}, \mathcal{O}_{c}$, and $\mathcal{O}_{b}$ are not reflexive.

Proof. Since $\mathcal{O}_{k}$ cannot be the dual space of any normed space, it follows that $\mathcal{O}_{k}$ is not reflexive. Thus the other two sequence spaces are also not reflexive.

Acknowledgement. We would like to thank the referees for their comments. This research was supported by the Centre of Excellence in Mathematics, the Commission on Higher Education, Thailand. 


\section{References}

[1] G. Bennett, Schur multipliers, Duke Math. J. 44 (1977), no. 3, 603-639.

[2] P. Chaisuriya and S.-C. Ong, Absolute Schur algebras and unbounded matrices, SIAM J. Matrix Anal. Appl. 20 (1999), no. 3, 596-605.

[3] I. E. Leonard, Banach sequence spaces, J. Math. Anal. Appl. 54 (1976), no. 1, 245-265.

[4] L. Livshits, S.-C. Ong, and S.-W. Wang, Banach space duality of absolute Schur algebras, Integral Equations Operator Theory 41 (2001), no. 3, 343-359.

[5] S.-C. Ong, On the Schur multiplier norm of matrices, Linear Algebra Appl. 56 (1984), $45-55$.

[6] J. Schur, Bemerkungen Theorie der beschranken Bilineärformen mit unendlich vielen Veränder lichen, J. Reine Angew. Math. 140 (1911), 1-28.

JiTTI RAKBUD

Department of Mathematics

FACUlTy OF SCIENCE

SILPAKORN UNIVERSITY

NAKORN PATHOM, 73000, Thailand

AND

Centre of Excellence in Mathematics

CHE, Si Ayutthaya Rd., Bangkok, 10400, Thailand

E-mail address: jitti@su.ac.th

Sing-CheOng Ong

Department of Mathematics

Central Michigan University

Mount Pleasant, MI 48858, USA

E-mail address: ong1s@cmich.edu 